\title{
Caracterización fenotíPICA de genotipos de dos POBLACIONES NATURALES DE SORGHASTRUM PELLITUM (PoACEAE) DEL SUDESTE BONAERENSE
}

\author{
PHENOTYPIC CHARACTERIZATION OF GENOTYPES OF TWO NATURAL \\ POPULATIONS OF SORGHASTRUM PELLITUM (POACEAE) FROM SOUTHEASTERN \\ Buenos Aires \\ María L. Echeverría ${ }^{1 *}$ (D), Gabriela A. Leofanti ${ }^{1}$ (D) Gisele E. Sanchez ${ }^{1}$ y \\ María. M. Echeverría ${ }^{1}$
}

1. Universidad Nacional de Mar del Plata, Facultad de Ciencias Agrarias, Balcarce, Buenos Aires, Argentina.

*mlecheverria@mdp.edu.ar

Citar este artículo

ECHEVERRÍA, M. L., G. A. LEOFANTI, G. E. SANCHEZ \& M. M. ECHEVERRÍA. 2021. Caracterización fenotípica de genotipos de dos poblaciones naturales de Sorghastrum pellitum (Poaceae) del sudeste bonaerense. Bol. Soc. Argent. Bot. 56: 513-531.

DOI: https://doi. org/10.31055/1851.2372.v56. n4.33811

Recibido: 6 Jul 2021

Aceptado: 25 Oct 2021

Publicado impreso: 20 Dic 2021

Editora: Paola Gaiero (D)

ISSN versión impresa 0373-580X

ISSN versión on-line 1851-2372

\section{SUMMARY}

Background and aims: Sorghastrum pellitum is a potentially ornamental grass species native to South America. Due to the demand for new native species by the floriculture market it is important to know if plants of this taxon can thrive under cultivation conditions and if they present variability for vegetative and/or reproductive attributes that make it possible to select genotypes to include in the market. The objective of this work was to characterize genotypes derived from two populations of $S$. pellitum that grow in mountainous areas of the southeastern Tandilia Hills (Buenos Aires Province, Argentina).

M\&M: Six genotypes of each population were used to obtain clones that were transferred to pots and grown outdoors. Variables related to transplant survival, morphology and phenology were recorded in two successive growing seasons.

Results: Intra- and inter-population variability was detected for transplant survival, morphological characters, duration of phenological stages and flowering time.

Conclusions: The implantation capacity under cultivation conditions and the morphological and phenological variability detected in this work suggest that this species could be included in breeding programs to obtain cultivars for subsequent use in landscaping. On the other hand, differences found between what was recorded in this work and what is found in the literature highlight the need to analyze a large number of specimens from different areas of its distribution when making taxonomic descriptions of this species, and to consider the existence of interspecific hybrids.

\section{KEY WORDS}

Biodiversity, conservation, native plant genetic resources, ornamental grasses, phenology, plant breeding.

\section{RESUMEN}

Introducción y objetivos: Sorghastrum pellitum es una Gramínea nativa de Sudamérica de gran potencial ornamental. Debido a la demanda de nuevas especies por el mercado floricultor, resulta importante conocer si prospera en condiciones de cultivo y si presenta variabilidad para los atributos vegetativos y/o reproductivos, que posibiliten la selección de genotipos para el mercado. El objetivo de este trabajo fue caracterizar genotipos de dos poblaciones de $S$. pellitum provenientes de áreas serranas del sudeste del Sistema Serrano de Tandilia (Buenos Aires, Argentina).

M\&M: Se utilizaron seis genotipos de cada población y se obtuvieron clones que se dispusieron en macetas al aire libre. En dos estaciones de crecimiento sucesivas se evaluaron variables relacionadas a la supervivencia al trasplante, morfología y fenología.

Resultados: Se registró variabilidad intra- e inter-poblacional en la supervivencia al trasplante, así como en caracteres morfológicos, duración de las etapas fenológicas y épocas de floración.

Conclusiones: La capacidad de implantación bajo cultivo y la variabilidad detectada, sugieren que esta especie podría incluirse en programas de mejoramiento genético, para la obtención de cultivares y posterior uso en planteos paisajísticos. Las diferencias encontradas entre lo registrado en este trabajo y la bibliografía, ponen en evidencia que al realizar descripciones taxonómicas de $S$. pellitum es necesario analizar un gran número de ejemplares, provenientes de las distintas áreas de su distribución, y considerar la existencia de híbridos interespecíficos.

\section{Palabras claves}

Biodiversidad, conservación, gramíneas ornamentales, mejoramiento genético vegetal, fenología, recursos fitogenéticos nativos. 


\section{INTRODUCCIÓN}

La demanda de nuevas especies vegetales y variedades ornamentales por parte del mercado floricultor local e internacional es creciente (Eynard et al., 2020); sin embargo, la oferta de materiales autóctonos de la República Argentina continúa siendo escasa y los productores locales deben recurrir al cultivo de materiales importados (Facciuto et al., 2019). Debido a la gran extensión del territorio y la multiplicidad climática, en la Argentina crecen de forma espontánea 10.221 especies de plantas vasculares, de las cuales se estima que más del $73 \%$ son nativas (Zuloaga et al., 2019), muchas de ellas de valor ornamental (Burgueño \& Nardini, 2018; Facciuto et al., 2019; Eynard et al., 2020). Por lo tanto, la inclusión de recursos fitogenéticos de origen nativo en el mercado floricultor podría representar una alternativa para satisfacer a productores y consumidores de plantas ornamentales. El uso de estos recursos fitogenéticos nativos con fines ornamentales presenta varios beneficios: los productores reducen los costos de adquisición de los cultivares y aumentan la oferta de cultivos novedosos; los consumidores economizan el mantenimiento y manejo de los espacios verdes y pueden recrear, al menos en parte, paisajes naturales (Burgueño \& Nardini, 2018; Facciuto et al., 2019; Eynard et al., 2020).

La demanda creciente de especies vegetales nativas responde a la existencia de una comunidad interesada en el uso de estas plantas para diseñar parques, jardines y/o corredores biológicos debido a los servicios ecosistémicos que estas brindan al ofrecer refugio y alimento para la fauna nativa y favorecer el mantenimiento de la biodiversidad. A su vez, con la constante fragmentación de ambientes naturales debido a la intensificación agrícola (Benton et al., 2003; Tscharntke et al., 2012), la importancia de los espacios verdes urbanos para la conservación de la biodiversidad aumenta (Goddard et al., 2010). En este contexto, la difusión y uso de plantas nativas en planteos paisajísticos contribuye al conocimiento de dichos recursos, así como de su cuidado, convirtiéndose en una estrategia para su conservación y valoración (Echeverría \& Camadro, 2020).

Ante la posibilidad de incorporar una nueva especie al mercado floricultor, es necesario caracterizar y evaluar diversas poblaciones con el fin de generar información de utilidad para los programas de mejoramiento vegetal (Ford-Lloyd \& Jackson, 1986). Resulta importante conocer su capacidad de adaptación fuera de su ambiente natural (ej. supervivencia al trasplante y establecimiento posterior), así como evaluar atributos vegetativos y/o reproductivos que posibiliten la selección de genotipos de alto valor ornamental (Echeverría \& Camadro, 2020). La detección de variabilidad genética para uno o más caracteres de interés permite la selección de genotipos que podrán ser empleados por el fitomejorador para la obtención de materiales que sean capaces de expresar varios caracteres deseables (Rimieri, 2017; Echeverría, 2018).

Las especies pertenecientes a la familia Gramíneas o Poaceae presentan notable capacidad de adaptación sustentada por su enorme diversidad morfológica, fisiológica y reproductiva (Nicora \& Rúgolo de Agrasar, 1987). Las Gramíneas ornamentales se caracterizan por presentar varas florales y hojas vistosas, así como un aspecto general de la planta muy llamativo gracias a sus tamaños, formas y colores, pudiendo presentar variaciones entre especies y entre variedades (Rúgolo de Agrasar \& Puglia, 2004; Nizzero, 2006; Clayton et al., 2021). Las plantas de este grupo se utilizan con éxito como ornamentales en espacios públicos, parques privados, jardines urbanos y suburbanos y pequeños espacios como patios, balcones y terrazas (Brookes, 2013). Esto se debe a sus características de longevidad, adaptación, rusticidad, bajo mantenimiento y a sus vistosas cualidades estéticas tales como color, forma y tamaño del follaje y de las inflorescencias (Rúgolo de Agrasar \& Puglia, 2004; Nizzero, 2006; Brookes, 2013). En la Argentina se han descrito 186 géneros y 1.167 especies, de las cuales 806 son nativas, 176 son endémicas y 168 son introducidas (Zuloaga et al., 2019). Esta riqueza, poco frecuente en otras partes del mundo de latitudes semejantes, resulta una gran fuente para la selección de taxones con potencial uso ornamental (Rúgolo de Agrasar \& Puglia, 2004).

Sorghastrum pellitum (Hack.) Parodi $(2 \mathrm{n}=2 \mathrm{x}=20)$, comúnmente conocida como "pasto colorado" o "pasto vaca" (Cabrera, 1970), es una especie nativa de Sudamérica, que se encuentra en Brasil, Paraguay, Uruguay y Argentina. Esta especie se adapta bien en zonas secas como sabanas, bancos secos y laderas de sierras, así como a zonas húmedas; es frecuente en campos, desde el nivel del mar hasta los 1000 msnm ocasionalmente, algunos individuos pueden 


\section{L. Echeverría et al. - Caracterización fenotípica de Sorghastrum pellitum (Poaceae)}

encontrarse a 2000 msnm (Dávila, 1988; Morrone \& Zuloaga, 2012). En la Argentina, el límite austral de su distribución está representado por el sur de las provincias de Buenos Aires y La Pampa. A su vez, es la única especie del género que se distribuye en el sudeste bonaerense (Morrone \& Zuloaga, 2012; Echeverría et al., 2017). Se trata de una especie estival, de ciclo perenne, que forma matas grandes y densas (Fig. 1A) (Parodi, 1930; Morrone \& Zuloaga, 2012). Sin embargo, debido a su valor forrajero, su uso ha sido reducido a áreas marginales donde se concentra la actividad ganadera y su aparente incapacidad para recolonizar áreas ha favorecido su merma en pastizales sobrepastoreados o sometidos a labranza mecánica, lo que ha representado una modificación irreversible y una pérdida de las poblaciones naturales (Ulrich et al., 2011).

Debido a la importancia de la inclusión de nuevas plantas nativas en el mercado floricultor y al potencial ornamental que presenta Sorghastrum pellitum, sumado al riesgo de pérdida de sus poblaciones en ambientes naturales, resulta importante conocer la capacidad de sobrevivencia de genotipos de esta especie fuera de su ambiente natural y la presencia de variabilidad para atributos vegetativos, reproductivos y fenológicos que posibiliten la selección de genotipos de alto valor ornamental. Por lo tanto, el objetivo del presente trabajo fue realizar una caracterización fenotípica inicial de genotipos de dos poblaciones naturales de áreas serranas del Sistema de Tandilia de S. pellitum en el sudeste bonaerense, considerando supervivencia y establecimiento en condiciones de cultivo, morfología y fenología con el fin de promover su incorporación en el mercado floricultor y contribuir así a la valoración y conservación de esta especie nativa.

\section{Materiales y MÉtodos}

\section{Materiales}

Se utilizaron individuos de dos poblaciones naturales de Sorghastrum pellitum, coleccionados en diciembre de 2016 en dos sitios pertenecientes al Sistema serrano de Tandilia (Pdo. de Balcarce, provincia de Buenos Aires) (Tabla 1, Fig. 1B). La población INTA proviene de un área serrana ubicada en la Estación Experimental Agropecuaria (EEA) del INTA de Balcarce (Fig. 1C) y la población BAR proviene de la Sierra La Barrosa (Fig. 1D).
Los ejemplares de referencia correspondientes a las poblaciones de este estudio se encuentran depositados en el herbario BAL (Thiers B., continuamente actualizado) según los registros 1019 y 3883.

\section{Ensayo a campo}

Se utilizaron seis plantas adultas (genotipos) de cada población que se mantenían desde el momento de su colección en un invernáculo de la Unidad Integrada Balcarce (UIB, conformada por la EEA INTA Balcarce y la Facultad de Ciencias Agrarias de la Universidad Nacional de Mar del Plata). En el otoño del 2017 se realizó la división de matas y se obtuvieron nueve clones de cada genotipo, a excepción de uno de BAR, para el que se obtuvieron siete clones, y de uno de BAR y otro de INTA para los que se obtuvieron ocho clones, por lo que en total se obtuvieron 51 plantas de la población BAR y 53 de la población INTA. A fin de que los clones presentaran un tamaño de mata parejas, cada clon tuvo aproximadamente cinco macollos. Las plantas obtenidas se dispusieron en macetas de 31 con un sustrato base constituido por tierra:turba:perlita (3:1:1, v/v/v) que fueron ubicadas en mayo de 2017 al aire libre en el campo, dentro del predio de la UIB ( $37^{\circ} 45^{\prime} 39,63^{\prime \prime} \mathrm{S} ; 58^{\circ} 17^{\prime} 59,75^{\prime \prime} \mathrm{O}$ ), siguiendo un diseño en bloques completos y aleatorizados con tres bloques (Fig. 2). Las unidades experimentales fueron parcelas de tres plantas (excepcionalmente cuatro parcelas contaron con dos plantas), correspondientes a un genotipo.

Las plantas permanecieron en el campo durante dos estaciones de crecimiento sucesivas: Estación de crecimiento 1 (E1), desde el trasplante, en mayo de 2017, a febrero de 2018, y Estación de crecimiento 2 (E2), desde marzo de 2018 a febrero de 2019. Se realizó el desmalezado manual y riego durante todo el período, y el corte de la mata a $10 \mathrm{~cm}$ para favorecer el macollaje, en junio del 2017 y mayo de 2018.

\section{Condiciones meteorológicas}

La caracterización de cada estación de crecimiento se realizó en base a registros diarios de precipitación y temperatura media de la Estación Meteorológica de la EEA Balcarce (EEA Balcarce INTA, 2021), calculándose Temperatura media mensual y Precipitación mensual acumulada para todos los meses de E1 y E2 así como la Precipitación total acumulada para cada estación de crecimiento. 
Bol. Soc. Argent. Bot. 56 (4) 2021
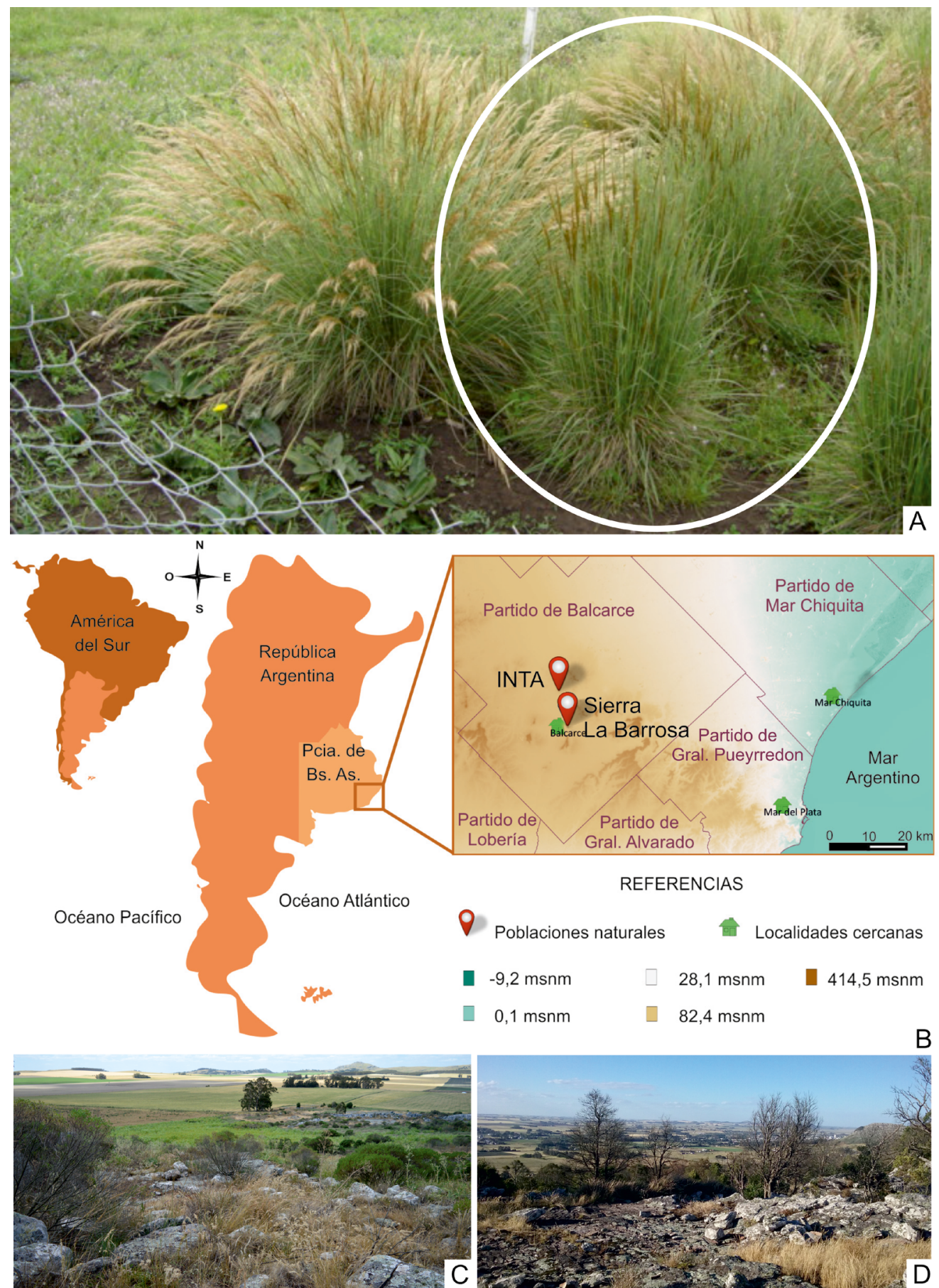

Fig. 1. A: Planta de Sorghastrum pellitum (señalada con un círculo blanco) creciendo junto a matas de Poa iridifolia Hauman en el Partido de Balcarce, Provincia de Buenos Aires. B: Mapa de América del Sur (marrón), con delimitación de la República Argentina (naranja) y de la Provincia de Buenos Aires (naranja claro) y detalle de una porción del sudeste bonaerense con la ubicación de los sitios de origen de las poblaciones naturales de S. pellitum consideradas en este estudio. C-D: Ambientes naturales de las poblaciones de S. pellitum de la EEA Balcarce INTA (INTA) y de la Sierra La Barrosa, respectivamente. 


\section{L. Echeverría et al. - Caracterización fenotípica de Sorghastrum pellitum (Poaceae)}

Tabla 1. Origen geográfico de las poblaciones de Sorghastrum pellitum del Sistema serrano de Tandilia empleados en este estudio.

\begin{tabular}{|c|c|c|c|}
\hline Población & Ubicación & $\begin{array}{l}\text { Coordenadas } \\
\text { geográficas }\end{array}$ & $\begin{array}{l}\text { Voucher y número de } \\
\text { colección de herbario }\end{array}$ \\
\hline INTA & $\begin{array}{l}\text { Cima del cerro adyacente al tambo del INTA de Balcarce, } \\
\text { Pdo. de Balcarce, provincia de Buenos Aires, Argentina }\end{array}$ & $\begin{array}{l}37^{\circ} 45^{\prime} 5,92^{\prime \prime} \mathrm{S}, 58^{\circ} 17^{\prime} \\
30,23^{\prime \prime} \mathrm{O} ; 182 \mathrm{msnm}\end{array}$ & ALO 881, BAL 1019 \\
\hline BAR & $\begin{array}{c}\text { Cima de sierra La Barrosa, Pdo. de Balcarce, } \\
\text { provincia de Buenos Aires, Argentina }\end{array}$ & $\begin{array}{l}37^{\circ} 53^{\prime} 7^{\prime \prime} \mathrm{S}, 58^{\circ} 16^{\prime} \\
\text { 3" O; } 305 \mathrm{msnm}\end{array}$ & OKA 5116, BAL 3883 \\
\hline
\end{tabular}

Registros en el ensayo a campo

Supervivencia al trasplante y establecimiento final: A partir de las plantas dispuestas en el ensayo al aire libre, en febrero 2018 se determinó el porcentaje de plantas que sobrevivieron al trasplante (PST) teniendo en consideración el número de plantas iniciales y el número de plantas muertas durante la primera estación de crecimiento (PM.E1). A su vez, en febrero 2019 se determinó el porcentaje de aquellas plantas establecidas al final del ensayo $(\mathrm{PEF})$, en relación al número inicial de plantas $\mathrm{y}$ el número de plantas muertas durante la primera $\mathrm{y}$

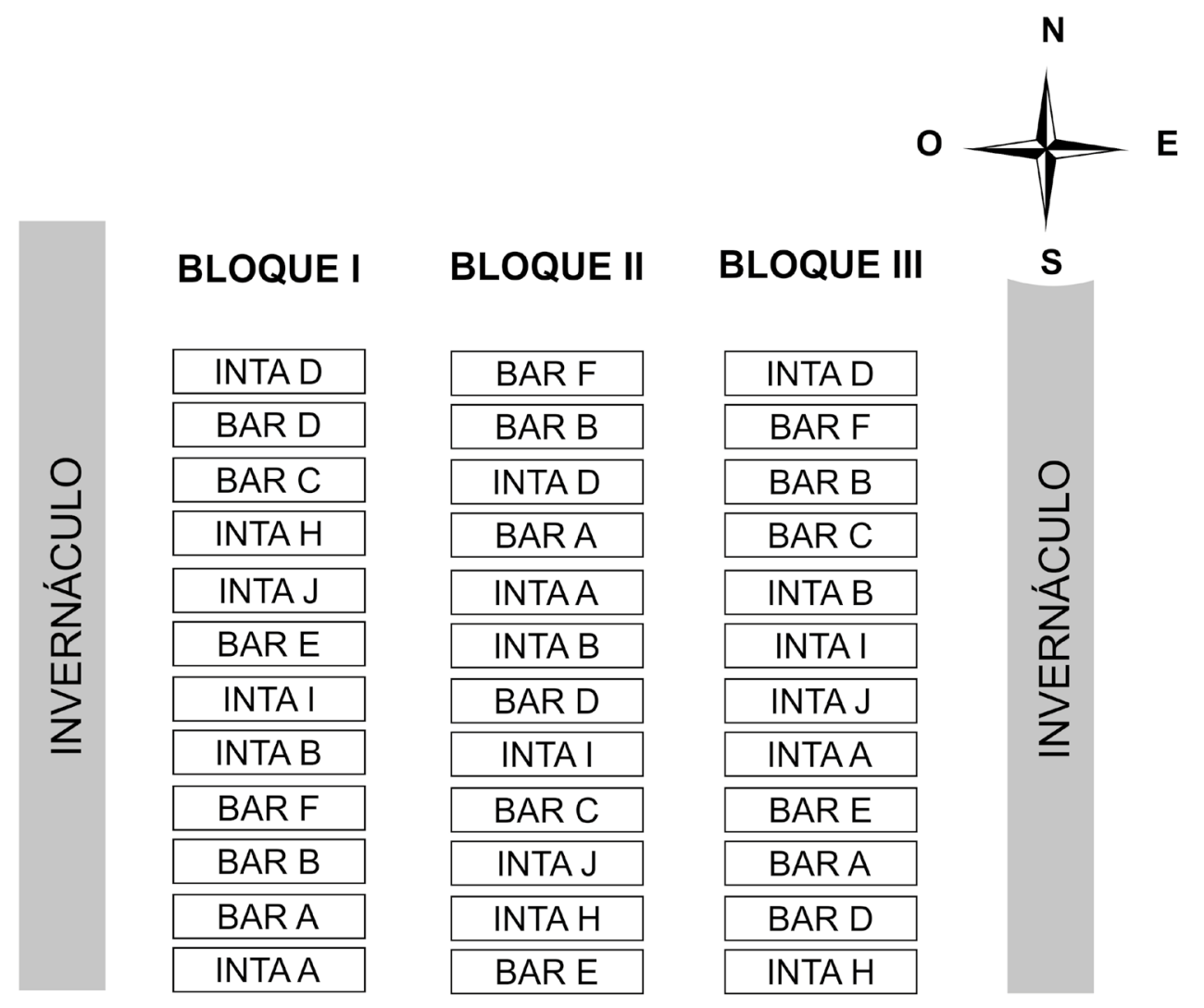

Fig. 2. Plano del ensayo de Sorghastrum pellitum con un diseño en bloques completos y aleatorizados, con tres repeticiones. Tratamientos: 6 genotipos pertenecientes a la población BAR $(A, B, C, D, E, F)$ y 6 genotipos pertenecientes a la población INTA $(A, B, D, H, I, J)$. 
segunda estación de crecimiento (PM.E1 y PM.E2, respectivamente).

Fenología: En la época primavero-estival de la E1 y la E2 se registraron las fechas de aparición de la primera y última panoja en cada planta florecida y así se calculó la duración de la floración de cada genotipo y de cada población.

Porcentaje de plantas florecidas: El porcentaje de plantas florecidas por genotipo se calculó teniendo en cuenta el número de plantas al inicio de cada estación de crecimiento.

\section{Caracteres morfológicos}

Número de panojas por planta: Se registró la aparición de nuevas panojas en todas las plantas del ensayo cada 4-7 días, desde el inicio al fin de la floración, en las dos estaciones de crecimiento. Se calculó el promedio por genotipo y por población de panojas nuevas/planta (PP_Nu) en cada fecha, y del total acumulado de panojas/planta (PP_T) al finalizar la floración.

Tamaño de mata: Se registró el diámetro de mata (DM) y la altura de mata (AM) en la E2, cada 20 días aproximadamente, desde junio de 2018 a enero de 2019 (11 fechas), en todas las plantas del ensayo. Para el DM se tuvo en cuenta la máxima extensión en ancho del follaje, medido como la distancia entre las hojas ubicadas en posición contrapuesta, mientras que la AM se midió desde la base de la mata hasta la máxima altura alcanzada por las hojas (Fig. 3A).

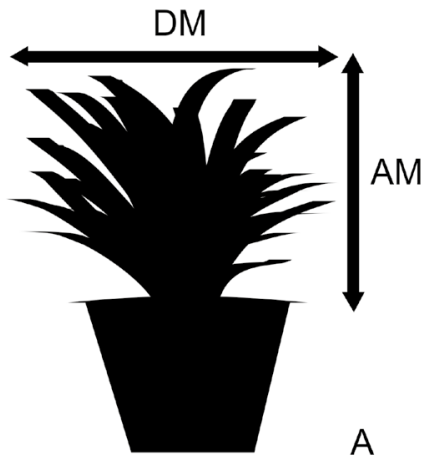

A

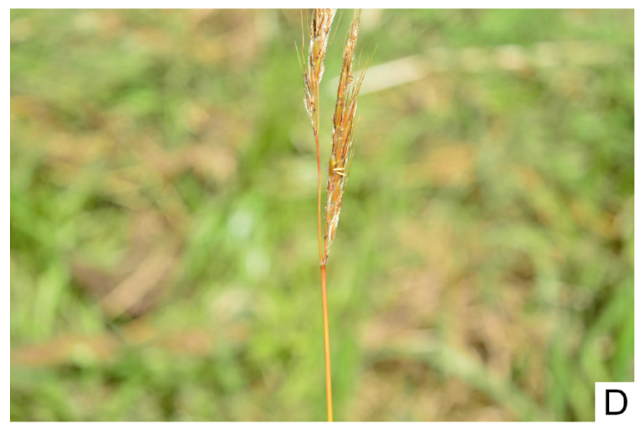

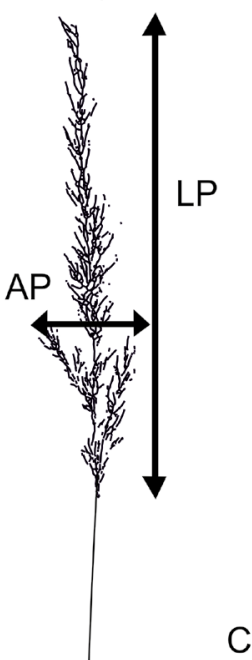

C

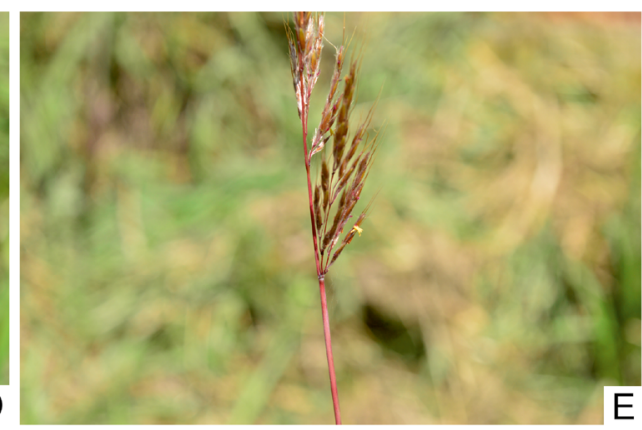

Fig. 3. Representación de las mediciones de A: altura (AM) y diámetro (DM) de mata, B: despeje de inflorescencia (flechas), C: longitud (LP) y ancho (AP) de panoja, y de los registros de $\mathbf{D}$ : pedúnculo de color anaranjado y E: pedúnculo de color violáceo en Sorghastrum pellitum. 


\section{L. Echeverría et al. - Caracterización fenotípica de Sorghastrum pellitum (Poaceae)}

Tamaño foliar: Se registró la longitud foliar (LF), desde la lígula hasta el ápice de la lámina, y ancho foliar en el ancho mayor de la lámina (AF). El registro se realizó sobre la última hoja desplegada de una planta por parcela seleccionada de manera aleatoria, por única vez al final de la E2, en los genotipos que estaban presentes en los tres bloques al día de la medición.

Despeje de la inflorescencia: En el período reproductivo de E2 se registró en todas las inflorescencias de todas las plantas la distancia entre el límite superior del canopeo y la base de la panoja cuando el pedúnculo alcanzó la máxima longitud (Fig. 3B). El despeje de cada individuo se calculó como el promedio de las distancias medidas a todas las panojas.

Tamaño de la panoja: Se registró la longitud de panoja (LP) y el ancho de la panoja (AP) en todas las panojas en ambas estaciones de crecimiento. En los individuos que presentaron más de una panoja, se calculó el promedio para ambas variables (Fig. 3C).

Presencia de antocianinas en vaina: Se registró de forma visual en todas las plantas del ensayo, en noviembre de 2017 y 2018.

Color de lámina: en la época estival se registraron los parámetros luminosidad (L), coordenadas rojo/verde (a) y coordenadas amarillo/azul (b) con colorímetro Konica Minolta CR-300, en una hoja bien desarrollada de cada una de las plantas que no presentaban signos de enfermedad ni de deshidratación.

Color del pedúnculo: Esta variable se estableció por apreciación visual como una variable cualitativa binaria, presentando las categorías: anaranjado (Fig. 3D) y violáceo (Fig. 3E). Se registró en cada planta florecida en ambas estaciones de crecimiento.

\section{Análisis estadísticos}

Se realizaron análisis de tipo univariado para las variables correspondientes al tamaño de mata, tamaño foliar y color de lámina. Los datos provenientes de las mediciones DM y AM se promediaron por parcela. Se eliminaron del análisis aquellos genotipos que perdieron todos los clones de una parcela. Los promedios así obtenidos fueron considerados para evaluar los efectos de genotipos y fechas de observación. El análisis se realizó bajo un modelo lineal con mediciones repetidas en el tiempo asignando los genotipos a las parcelas principales y las fechas de observación a las subparcelas. Se realizaron los Análisis de la Varianza (ANOVA) y, cuando la interacción entre genotipo y fecha resultó significativa $(\alpha=0,05)$, se continuó el análisis de la misma con un ANOVA por fecha. Ante la presencia de diferencias significativas, los promedios de los genotipos se compararon mediante la prueba de Tukey $(\alpha=0,05)$. Los datos provenientes de las mediciones de tamaño y color foliar se promediaron por parcela y se realizó un ANOVA. Cuando se detectaron diferencias significativas, los promedios de los genotipos se compararon mediante la prueba de Tukey $(\alpha=0,05)$. Todos los análisis se realizaron empleando el programa estadístico Infostat (Di Rienzo et al., 2016).

\section{Resultados}

Variables meteorológicas: temperatura media mensual y precipitaciones acumuladas

Los valores de temperatura media mensual fueron superiores para la E1 en los meses de invierno con respecto a la $\mathrm{E} 2$, siendo para junio, julio y agosto de $9,8^{\circ} \mathrm{C}, 9,9^{\circ} \mathrm{C}$ y $11,4^{\circ} \mathrm{C} v s .8,3^{\circ} \mathrm{C}, 8,3^{\circ} \mathrm{C}$ y $9,6^{\circ} \mathrm{C}$, respectivamente. En los meses restantes se registraron temperaturas medias mensuales similares para ambas estaciones de crecimiento (Fig. 4A).

La precipitación total acumulada para la E1 y para la E2 fue de $907,3 \mathrm{~mm}$ y $1143,9 \mathrm{~mm}$, respectivamente. Por otro lado, en la E1, los valores de precipitación mensual acumulada fueron superiores en la mayoría de los meses del período otoño-inverno-primaveral, pero en los meses del verano la situación fue la inversa (Fig. 4B). Además, el mayor registro de precipitación mensual acumulada ocurrió en abril $(250 \mathrm{~mm})$ para $\mathrm{E} 1 \mathrm{y}$ en noviembre $(150 \mathrm{~mm})$ para E2.

Supervivencia al trasplante y establecimiento final

Del total de plantas que fueron trasplantadas para conformar el ensayo a campo, un número variable según el genotipo permaneció en el campo hasta el final de ambas estaciones de crecimiento (PEF, plantas establecidas al final). Algunas plantas murieron durante la E1 (PM.E1) mientras que otras lo hicieron durante la E2 (PM.E2). Las plantas que permanecieron hasta por lo menos el inicio de la E2 se consideraron sobrevivientes al trasplante (PST). En la Figura 5A, se muestran los porcentajes de estas cuatro variables, por población y por genotipo. 

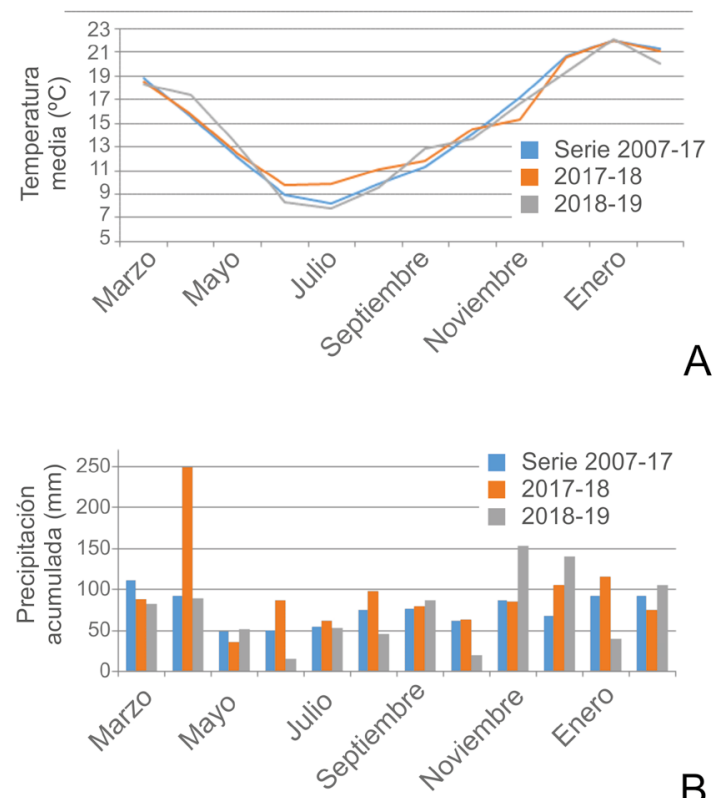

Fig. 4. Temperatura media mensual $\left({ }^{\circ} \mathrm{C}\right)(\mathrm{A})$ y precipitación mensual acumulada $(\mathrm{mm})$ (B) para los períodos de 2017-18 y 2018-19 y para la serie 2007-17 en la UIB.

Al comparar las poblaciones BAR e INTA se observó que la primera presentó el mayor porcentaje de PST (70,6\% vs. 54,7\%) y de PEF $(52,9 \%$ vs. $45,3 \%)$. La diferencia para esta última variable fue menor debido a que BAR presentó un mayor porcentaje de PM.E2 (17,6\% vs. 9,4\%).

$\mathrm{Al}$ analizar los genotipos de forma individual se observó que BAR B presentó el mayor porcentaje de PST en BAR B $(85,7 \%)$ y el menor en BAR C e INTA D (37,5\%). El genotipo BAR B presentó el mayor porcentaje de PEF ya que no tuvo pérdida de plantas durante la E2 y los genotipos INTA A e INTA D presentaron los porcentajes más bajos, siendo en ambos casos inferiores a $25 \%$.

\section{Fenología}

Todos los genotipos de la población INTA florecieron en ambas estaciones de crecimiento mientras que el $50 \%$ de los genotipos de BAR floreció en la E1 y el 100\% en la E2 (Fig. 5B). Las dos poblaciones iniciaron su floración a fines de noviembre en ambas estaciones de crecimiento. Sin embargo, la fecha de finalización de esta etapa, así como la duración de la misma presentaron variaciones entre poblaciones y entre estaciones de crecimiento. Las plantas de la población BAR finalizaron la floración a mediados de diciembre en la E1 y principios de enero en la E2 duplicando así la duración de esta fase reproductiva, que fue de 15 días para la primera y de 39 días para la segunda. Para la población INTA se observó menor variación entre estaciones de crecimiento ya que en ambas la floración finalizó a principios de enero y la duración fue de 37 y 39 días en la E1 y la E2, respectivamente.

$\mathrm{Al}$ considerar cada genotipo de forma individual se detectaron amplias diferencias entre ellos, tanto en las fechas de inicio y finalización de la floración como en la duración de esta fase, pero en general se observó un adelanto de la floración en la E2 con respecto a la estación anterior. Los genotipos que más variaron entre estaciones fueron INTA I y BAR $\mathrm{F}$ que adelantaron la floración 18 días en la E2.

En la E1 los genotipos BAR A e INTA J fueron los que florecieron primero (29/11/18); mientras que BAR F e INTA B, H e I fueron los últimos en iniciar esta etapa y lo hicieron dos semanas más tarde (13/12/18). Por otra parte, los genotipos BAR A e INTA A y J fueron los primeros en finalizar esta etapa $(6 / 12 / 18)$ mientras que INTA $H$ fue el último, casi un mes después (4/01/19). Para los genotipos de la población BAR la floración se extendió entre uno (BAR F) y ocho días (BAR A y E), mientras que para los genotipos de la población INTA duró entre uno (INTA A y B) y 23 días (INTA H).

En la E2 el inicio de la floración se concentró en la última semana de noviembre para todos los genotipos, excepto para BAR C que emitió su primera panoja a fines de diciembre. Por otro lado, BAR A y B fueron los primeros en finalizar la floración (25/11/18), mientras que los genotipos INTA J y BAR E fueron los últimos (02/01/19). Para ambas poblaciones, la floración se extendió entre 1 (BAR A, B y C, INTA A y D) y 39 días (BAR E e INTA J). Es necesario aclarar que debido a que la duración de la floración se realizó en base a la aparición de nuevas panojas, aquellas plantas que presentaron una duración de un día fue porque en ellas se registraron nuevas panojas en una sola fecha, sin embargo, esas panojas permanecieron en la planta varios días.

En ambas estaciones de crecimiento el promedio de la duración de la floración fue mayor para la población INTA con respecto a BAR, con valores de 9,3 vs. 5,7 días en la E1 y 14 vs. 9 días en la E2. 

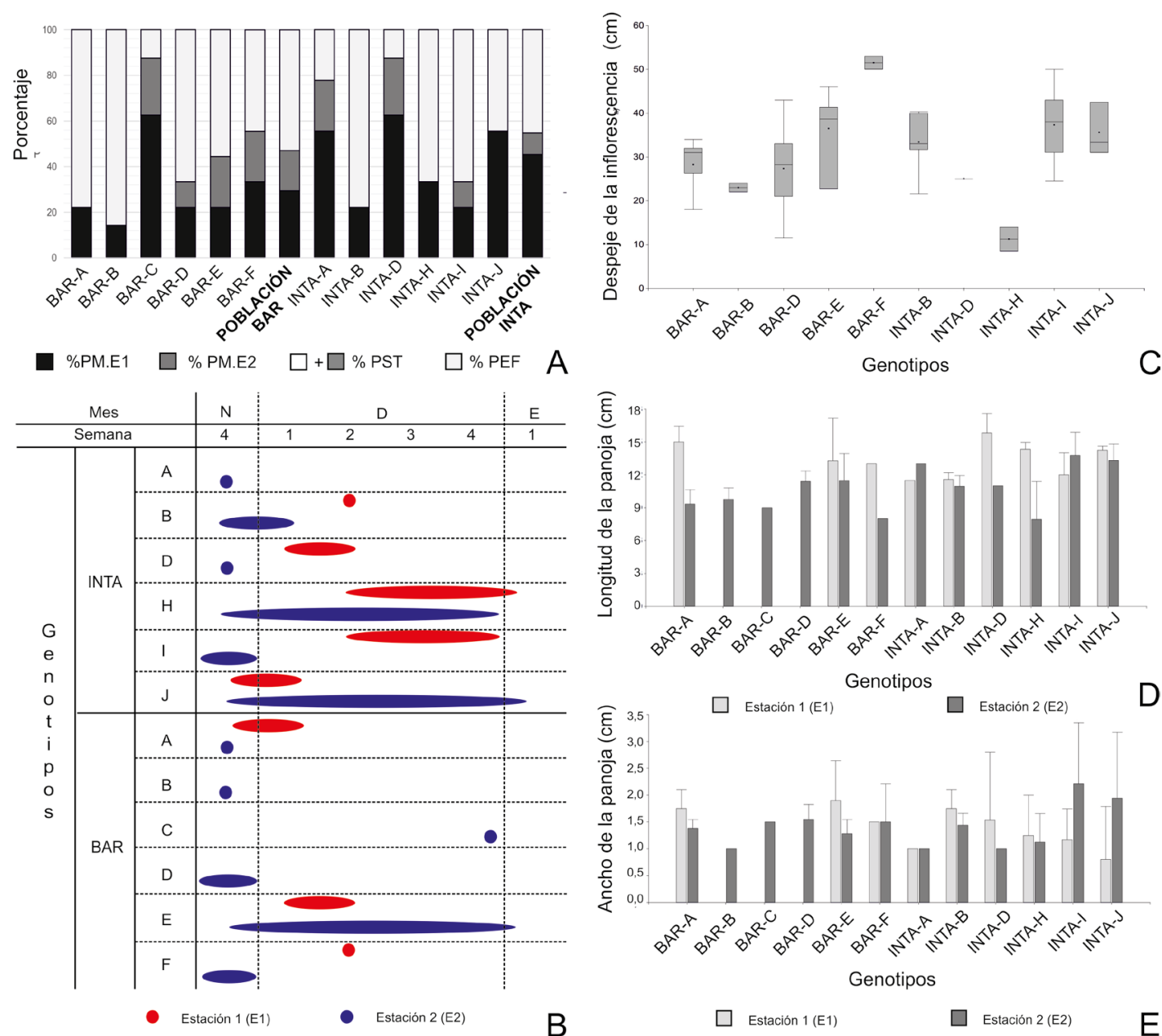

Fig. 5. A: Porcentajes de plantas muertas (PM.E1 y PM.E2), de plantas sobrevivientes al transplante (PST) y de plantas establecidas (PEF) en genotipos pertenecientes a dos poblaciones de Sorghastrum pellitum, INTA y BAR, al ser cultivados ex situ en Balcarce, Buenos Aires; B: Período de floración para genotipos de dos poblaciones de $S$. pellitum (INTA y BAR) cultivados en Balcarce, Buenos Aires, en dos estaciones de crecimiento, E1 (rojo) y E2 (azul); C: Diagramas de caja para la variable despeje de inflorescencia (cm) de genotipos de dos poblaciones de S. pellitum (INTA y BAR) cultivados ex situ en Balcarce, Buenos Aires; D: Longitud de panoja y E: ancho de panoja para genotipos de dos poblaciones de S. pellitum (INTA y BAR) cultivadas ex situ en Balcarce, Buenos Aires, en dos estaciones de crecimiento (E1 y E2). Las barras señalan desvío estándar. Ref.: Estaciones de crecimiento (E), E1: mayo de 2017 a febrero de 2018; E2: marzo de 2018 a febrero de 2019.

\section{Porcentaje de plantas florecidas}

Las dos poblaciones presentaron porcentajes de plantas florecidas similares, tanto en la E1 como en la E2 y en general los valores fueron superiores en la E2, tanto a nivel poblacional como a nivel de genotipo individual (Tabla 2). En la E1 el menor porcentaje de plantas florecidas lo presentaron los genotipos BAR F e INTA A $(11,1 \%)$, mientras que el mayor porcentaje correspondió a BAR E $(55,6 \%)$. Por otro lado, en la E2 el menor porcentaje lo presentó INTA A (25\%); mientras que el mayor porcentaje lo presentó un genotipo de la misma población, INTA B, para el cual florecieron todas las plantas (Tabla 2). 
Tabla 2. Porcentaje de plantas florecidas por genotipo en dos poblaciones de Sorghastrum pellitum, INTA y BAR, al ser cultivados ex situ en Balcarce, Buenos Aires, durante dos estaciones de crecimiento, E1 (mayo de 2017 a febrero de 2018)

y E2 (marzo de 2018 a febrero de 2019).

\begin{tabular}{|ccc|}
\hline Genotipol & Porcentaje de plantas florecidas \\
Población & E1 & E2 \\
\hline INTAA & 11,1 & 25 \\
INTA B & 22,2 & 100 \\
INTA D & 37,5 & 33,3 \\
INTA H & 33,3 & 33,3 \\
INTA I & 33,3 & 71,4 \\
INTA J & 22,2 & 75 \\
$\overline{\text { X INTA }}$ * & 26,6 & 56,4 \\
BAR A & 22,2 & 71,4 \\
BAR $B^{* *}$ & - & 33,3 \\
BAR C $^{* *}$ & - & 33,3 \\
BAR D $^{* *}$ & - & 71,4 \\
BAR E $^{*}$ & 55,6 & 57,1 \\
BAR F $_{\bar{X}}$ BAR & 11,1 & 33,3 \\
\hline
\end{tabular}

Ref. * Los porcentajes de plantas en flor fueron calculados sobre el número de plantas al inicio de cada estación de crecimiento. Los promedios poblacionales se calcularon a partir de los porcentajes individuales de los genotipos florecidos. ** Genotipos que no florecieron en E1.

\section{Caracteres morfológicos}

Número de panojas por planta: En general se observó que la aparición de nuevas panojas estuvo concentrada en las primeras dos semanas de diciembre en la E1 y la última semana de noviembre en la E2.

En la E1, los genotipos de la población INTA que registraron el máximo promedio fueron INTA B e INTA $\mathrm{H}$ el día 13/12/18 con dos PP_Nu, mientras que para la población $\mathrm{BAR}$, ningún genotipo registró más de una $\mathrm{PP} \mathrm{Nu}$ en ninguna fecha. Por otro lado, en la E2 los genotipos que presentaron los mayores promedios de esta variable fueron INTA $\mathrm{J}$ el $25 / 11 / 18$ e INTA $\mathrm{H}$ el $30 / 11 / 18$, con cuatro PP_Nu y BAR E, el 30/11/18 con seis PP_Nu.
En cuanto PP T, ambas poblaciones presentaron mayores valores promedio en la E2 con respecto a E1, siendo 2,1 vs. 1,3 para INTA, y 2 vs. 1 para BAR. En cuanto al comportamiento individual de los genotipos, en la E1 BAR A, E y F, los únicos en florecer de esta población, presentaron un promedio de una PP_T. En la E2, en cambio, BAR E registró el máximo valor promedio de $\mathrm{PP}$ T (4) y BAR C y BAR F el mínimo (1). Por otro lado, para la población INTA, en la E1 el genotipo INTA B presentó el máximo promedio de PP_T (2), e INTA A, D, I y J registraron el mínimo (1). En la E2, al igual que en la primera estación de crecimiento, el genotipo INTA $\mathrm{B}$ fue el que registró el máximo promedio de PP_T (3), y el mínimo se registró en los genotipos INTĀA e INTA D (1).

Color de lámina: Para el análisis de las variables registradas con el colorímetro se eliminó el genotipo INTA D ya que, debido a la pérdida de plantas, no se encontraba representado en todos los bloques.

Tamaño de mata: En general se observó que en la medida que avanzaba la estación de crecimiento todos los genotipos aumentaron tanto la altura como el diámetro de la mata (Tabla 3).

Para ambas variables se observó interacción genotipo $\times$ fecha $(p<0,001)$, por lo que para estas variables se procedió a analizar el comportamiento de los genotipos en cada fecha de muestreo. Estos análisis evidenciaron que las diferencias entre genotipos fueron en aumento hasta ser estadísticamente significativas en las últimas fechas de registro. En el caso de AM, sólo se detectaron diferencias significativas en las últimas dos fechas (Tabla 3) donde los promedios más bajos fueron obtenidos por el genotipo BAR F $(19,8 \mathrm{~cm}$ y $21,2 \mathrm{~cm})$ y los más altos por el genotipo INTA I $(30,9 \mathrm{~cm}$ y $34,2 \mathrm{~cm})$ el $26 / 12 / 2018$ y 16/01/2019, respectivamente. Por otro lado, para DM se detectaron diferencias significativas en las últimas tres fechas (Tabla 3), en las cuales los promedios más altos los presentó el genotipo BAR E. En la última fecha, en la que todos los genotipos alcanzaron sus máximos valores, BAR E presentó un valor medio cercano a los $40 \mathrm{~cm}$ y se diferenció de forma significativa de dos genotipos de la misma población, BAR $\mathrm{B}$ y $\mathrm{BAR} \mathrm{F}$, que presentaron un promedio menor a $27 \mathrm{~cm}$.

Tamaño foliar: Para las variables LF y AF no se registraron diferencias significativas entre los genotipos. En cuanto a LF, la media general fue de $16,1 \mathrm{~cm}$, con un $\mathrm{CV}$ de $28,7 \%$ y tanto el 


\section{L. Echeverría et al. - Caracterización fenotípica de Sorghastrum pellitum (Poaceae)}

Tabla 3. Altura de mata (AM) y diámetro de mata (DM) de de nueve genotipos pertenecientes a dos poblaciones de Sorghastrum pellitum, INTA y BAR, al ser cultivados ex situ en Balcarce, Buenos Aires. Valores promedio por genotipo en once fechas.

\begin{tabular}{|c|c|c|c|c|c|c|c|c|c|c|c|c|}
\hline \multirow{2}{*}{ Variable } & \multirow{2}{*}{ Genotipo } & \multicolumn{11}{|c|}{ Fecha } \\
\hline & & 15-Jun & 5-Jul & 31-Jul & 21-Aug & 7-Sep & 28-Sep & $16-$-Oct & 5-Nov & 30-Nov & 26-Dec & 16-Jan \\
\hline \multirow[t]{9}{*}{ AM (cm) } & BAR A & 10,2 & 10,4 & 10,6 & 12,3 & 14,8 & 19,1 & 20,2 & 22,3 & 22,7 & $23,8 \mathrm{abc}$ & $26,1 \mathrm{ab}$ \\
\hline & BAR B & 9,6 & 10 & 10,3 & 10,9 & 14,1 & 17,3 & 17,8 & 19,3 & 21 & $22,8 \mathrm{ab}$ & $25,3 \mathbf{a}$ \\
\hline & BAR D & 10,1 & 10,6 & 11,6 & 12,3 & 16 & 18,8 & 20,7 & 20,8 & 20,9 & 26,9 abc & 27,4 ab \\
\hline & BAR E & 10,1 & 10,9 & 11,7 & 12,1 & 14,8 & 21,5 & 23,5 & 27 & 27,3 & 28,2 bc & $28,2 a b$ \\
\hline & BAR F & 9,7 & 10,5 & 10,8 & 11 & 12,4 & 15 & 16,5 & 17 & 19 & $19,8 \mathbf{a}$ & $21,2 \mathbf{a}$ \\
\hline & INTA B & 10,3 & 10,6 & 10,8 & 11,8 & 15,8 & 19,9 & 23 & 24 & 24,4 & $25,2 \mathrm{abc}$ & $28,1 \mathrm{ab}$ \\
\hline & INTA H & 10,5 & 11,5 & 12,1 & 12,6 & 14,2 & 18,5 & 19,3 & 20,6 & 21,2 & $23,7 \mathrm{abc}$ & 25,5 a \\
\hline & INTA & 10,4 & 11,4 & 12,3 & 12,6 & 15,4 & 19,3 & 21,7 & 23,4 & 7,4 & c & 34,2 b \\
\hline & INTA J & 10,3 & 1 & 12,3 & 3 & 16 & 18,3 & 21 & 7 & 7 & $a b$ & $21,7 \mathrm{a}$ \\
\hline \multirow[t]{9}{*}{ DM (cm) } & BAR A & 14,6 & 14,4 & 14,7 & 15,1 & 15,3 & 19,4 & 19,9 & 19,9 & 20,8 a & $23,9 \mathbf{a}$ & 26,8 ab \\
\hline & BAR B & 11,5 & 10,9 & 11,1 & 12,1 & 13 & 15,7 & 16,9 & 17,9 & 20,2 a & $24,3 \mathbf{a}$ & $25,4 \mathbf{a}$ \\
\hline & BAR D & 11,5 & 12 & 12,1 & 12,5 & 14,3 & 16,9 & 19,4 & 20 & 22,8 ab & $25,8 \mathrm{ab}$ & 28,4 ab \\
\hline & BAR E & 13,4 & 13,4 & 14,9 & 15,2 & 15,7 & 22,2 & 23,5 & 24,5 & $31,3 \mathbf{b}$ & $33,8 \mathbf{b}$ & $39,3 \mathbf{b}$ \\
\hline & BAR F & 13,1 & 13,7 & 13,8 & 14,2 & 15,8 & 17,4 & 18,2 & 19,6 & 23,0 ab & $25,2 \mathrm{ab}$ & $26,3 \mathbf{a}$ \\
\hline & INTA B & 13,3 & 14 & 15,2 & 15,3 & 16,9 & 18,8 & 21,2 & 21,8 & 24,1 ab & $28,2 \mathrm{ab}$ & $31,4 \mathbf{a b}$ \\
\hline & INTA H & 11,9 & 13,3 & 14,1 & 14,8 & 15,8 & 18,6 & 19,8 & 20,1 & 23,7 ab & $29,1 \mathrm{ab}$ & 30,8 ab \\
\hline & INTA I & 10,4 & 10,8 & 11,5 & 11,7 & 13,6 & 15,6 & 16,3 & 16,6 & $20,4 \mathbf{a}$ & $24,1 \mathrm{a}$ & $34,7 \mathrm{ab}$ \\
\hline & INTA J & 13,3 & 14,3 & 13,7 & 13,7 & 14,7 & 18,3 & 19,3 & 20,7 & $25,0 a b$ & $26,3 \mathbf{a b}$ & $29,3 \mathbf{a b}$ \\
\hline
\end{tabular}

promedio mínimo como el máximo correspondieron a genotipos de la población BAR (BAR B: $13,1 \mathrm{~cm}$ y BAR E: $18,7 \mathrm{~cm})$. El genotipo que presentó mayor variabilidad fue BAR E, con un desvío estándar de $6,9 \mathrm{~cm}$, y el que presentó menor variabilidad fue BAR B, con un desvío estándar de $2,1 \mathrm{~cm}$ (Tabla 4). Los promedios poblacionales de LF fueron muy similares, siendo de $16 \mathrm{~cm}$ para BAR y de $16,3 \mathrm{~cm}$ para INTA.

Para AF, la media general fue de $0,47 \mathrm{~cm}$, con un $\mathrm{CV}$ de $25,4 \%$, y tanto las medias poblacionales como las de los genotipos fueron muy similares, entre 0,4 y $0,5 \mathrm{~cm}$. El genotipo que presentó mayor variabilidad fue BAR D, con un desvío de $0,21 \mathrm{~cm}$, y el que presentó menor variabilidad fue BAR A, con un desvío de $0 \mathrm{~cm}$ (Tabla 4).

Despeje de la inflorescencia: Esta variable presentó valores medios cercanos a los $30 \mathrm{~cm}$. A su vez se observó que los mayores valores tanto a nivel poblacional como a nivel de genotipo correspondieron a BAR. La diferencia entre los promedios poblacionales fue menor a $5 \mathrm{~cm}$ mientras que la diferencia entre genotipos fue de más de $40 \mathrm{~cm}$, siendo el valor mínimo para INTA H $(11,3 \mathrm{~cm})$ y el máximo para BAR $\mathrm{F}(51,5$ $\mathrm{cm})$. A su vez, se observaron diferencias entre plantas dentro de cada genotipo y el que presentó mayor variabilidad fue BAR D (D.E.= 11,93 cm) (Fig. 5C).

Longitud de panoja: Anivel poblacional se observaron valores medios de LP similares para BAR e INTA en E1 $(13,8 \mathrm{~cm} v s .13,3 \mathrm{~cm})$ pero no en la E2, en donde INTA presentó mayor promedio que BAR $(12,1 \mathrm{~cm}$ vs. 9,9 cm). En la E1 el menor y el mayor promedio por genotipo correspondieron a la población INTA (INTA A: $11,5 \mathrm{~cm}$ e INTA D: $15,8 \mathrm{~cm}$ ). Por su parte en E2, el valor mínimo se registró en BAR F $(8 \mathrm{~cm})$ y el máximo en INTA I $(13,7 \mathrm{~cm})$ (Fig. 5D). A su vez, se observaron variaciones entre mediciones dentro de un mismo genotipo. El genotipo BAR E fue el que presentó mayor variabilidad para LP en la E1 (D.E. $=3,8 \mathrm{~cm}$ ) y el INTA-H quien lo presentó en la $\mathrm{E} 2$ (D.E. $=3,4 \mathrm{~cm}$ ). 


\begin{tabular}{|c|c|c|c|c|}
\hline $\begin{array}{r}\text { Tabla 4. V } \\
\text { (min.- m } \\
\text { foliar (AF) } \\
\text { de dos } \\
\text { INTA y BA }\end{array}$ & $\begin{array}{r}\text { alores promed } \\
\text { ax.) y desvío } \\
\text { longitud folit } \\
\text { ooblaciones o } \\
\text { R, al ser culti } \\
\text { Buen }\end{array}$ & $\begin{array}{l}\text { dio }(\bar{x} \\
\text { están } \\
\text { ar (LF } \\
\text { le So } \\
\text { ivados } \\
\text { os Air }\end{array}$ & $\begin{array}{l}\text {, mínimos y má } \\
\text { dar (D.E.) de an } \\
\text { ) para siete gen } \\
\text { ghastrum pellitu } \\
\text { ex situ en Balc } \\
\text { es. }\end{array}$ & $\begin{array}{l}\text { ximos } \\
\text { cho } \\
\text { otipos } \\
\text { Im, } \\
\text { arce, }\end{array}$ \\
\hline Genotipo / & $\mathrm{AF}(\mathrm{cm})$ & & LF (cm) & \\
\hline Población & $\bar{x}$ (mín.- máx.) & D.E. & $\bar{x}$ (mín.- máx.) & D.E. \\
\hline BAR A & $0,5(0,5)$ & 0 & $17,2(10,6-20,7)$ & 5,69 \\
\hline BAR B & $0,4(0,3-0,5)$ & 0,12 & $13,1(11,0-15,2)$ & 2,1 \\
\hline BAR D & $0,5(0,3-0,7)$ & 0,21 & $16,9(10,0-22,6)$ & 6,39 \\
\hline BAR E & $0,5(0,4-0,7)$ & 0,15 & $18,7(12,7-26,2)$ & 6,86 \\
\hline BAR F & $0,5(0,4-0,5)$ & 0,05 & $14,0(10,2-17,7)$ & 3,75 \\
\hline BAR & 0,5 & & 16 & \\
\hline INTA H & $0,4(0,4-0,5)$ & 0,06 & $14,6(11,3-16,5)$ & 2,89 \\
\hline INTA I & $0,4(0,3-0,5)$ & 0,1 & $18(13,1-22,8)$ & 4,85 \\
\hline INTA & 0,4 & & 16,3 & \\
\hline
\end{tabular}

En cuanto al AP en la E1 se observó que en la población BAR el valor promedio de esta variable fue superior $(1,7 \mathrm{~cm})$ respecto al de la población INTA $(1,3 \mathrm{~cm})$ mientras que en E2 los valores de AP promedios fueron similares para ambas poblaciones (BAR: 1,4 cm; INTA: 1,5 cm). En ambas estaciones de crecimiento los valores más bajos fueron cercanos a $1 \mathrm{~cm}$ y los más altos variaron de entre $1 \mathrm{y} 4 \mathrm{~cm}$, aunque los genotipos que presentaron estos extremos fueron distintos entre estaciones (Fig. 5E). A su vez, se observaron variaciones entre mediciones dentro de un mismo genotipo, siendo el genotipo INTA $J$ el que presentó mayor variabilidad tanto en la E1 (D.E.= $1 \mathrm{~cm}$ ) como en la E2 (D.E. $=1,2 \mathrm{~cm}$ ). Cuatro de los genotipos de INTA en la E1 registraron valores mínimos menores a $0,5 \mathrm{~cm}$.

Presencia de antocianinas en vaina: La presencia de antocianinas en vaina fue variable, aunque en general predominó su ausencia. En algunos genotipos, como INTA B o BAR A, se observaron diferencias entre clones ya que algunos de ellos presentaron antocianinas en vaina y otros no.

Al realizar el análisis de la varianza, se observaron diferencias significativas entre genotipos para las variables "a" (coordenada rojo/ verde), y "b" (coordenada amarillo/azul) pero no para "L" (luminosidad) (Tabla 5).
Con respecto a la variable "a", los valores obtenidos siempre fueron negativos debido a la preponderancia del color verde para esta coordenada, mientras que para " $b$ " los valores fueron siempre positivos debido en este caso a la preponderancia del color amarillo (Fig. 6). Los genotipos BAR E e INTA A fueron los que presentaron los promedios más extremos para estos dos parámetros. BAR E presentó el valor medio más alto de "a" $(-10,8)$ y el más bajo de "b" $(17,9)$ que indican la presencia de los colores verde y amarillo menos intensos o puros respectivamente. Lo contrario se registró en el genotipo INTA A que presentó el valor medio más bajo de "a" (-15) y el más alto de "b" $(24,1)$ que indican la presencia de los colores verde y amarillo, más puros. Por último, el promedio general de L fue de 48 (Tabla 5, Fig. 6), el cual corresponde a un valor intermedio considerando que este parámetro varía de 0 a 100.

Color de pedúnculo: Para la variable color de pedúnculo, en E1 predominó el color anaranjado mientras que en E2 fue más frecuente la coloración violácea. En algunos casos se observó que clones de un mismo genotipo presentaban variación respecto al color.

Tabla 5. Color de lámina según las variables coordenadas rojo/verde ("a"), coordenadas amarillo/azul ("b") y luminosidad ("L") para genotipos de dos poblaciones de Sorghastrum pellitum (INTA y BAR) cultivadas en Balcarce, Buenos Aires. Valores promedio por genotipo. Letras iguales dentro de una misma variable indican diferencias no significativas, $p$-valor $>0,05$.

\begin{tabular}{|cccc|}
\hline Genotipos & "a" & $\begin{array}{c}\text { Variable } \\
\text { "b" }\end{array}$ & "L" \\
\hline BAR A & $-12,3 \mathrm{ab}$ & $19,2 \mathrm{ab}$ & 45,3 \\
BAR B & $-13,0 \mathrm{abc}$ & $20,6 \mathrm{ab}$ & 47,1 \\
BAR C & $-14,3 \mathrm{bcd}$ & $23,4 \mathrm{ab}$ & 49,5 \\
BAR D & $-13,4 \mathrm{abcd}$ & $22,5 \mathrm{ab}$ & 48,3 \\
BAR E & $-10,8 \mathrm{a}$ & $17,9 \mathrm{~b}$ & 48,6 \\
BAR F & $-14,6 \mathrm{~d}$ & $23,1 \mathrm{a}$ & 46,7 \\
INTA A & $-15,0 \mathrm{~d}$ & $24,1 \mathrm{a}$ & 50,6 \\
INTA B & $-13,3 \mathrm{abcd}$ & $23,3 \mathrm{ab}$ & 49,1 \\
INTA H & $-14,7 \mathrm{~cd}$ & $23,2 \mathrm{ab}$ & 47,1 \\
INTA I & $-13,4 \mathrm{bcd}$ & $21,7 \mathrm{ab}$ & 47,9 \\
INTA J & $-14,3 \mathrm{bcd}$ & $23,0 \mathrm{ab}$ & 47 \\
\hline
\end{tabular}



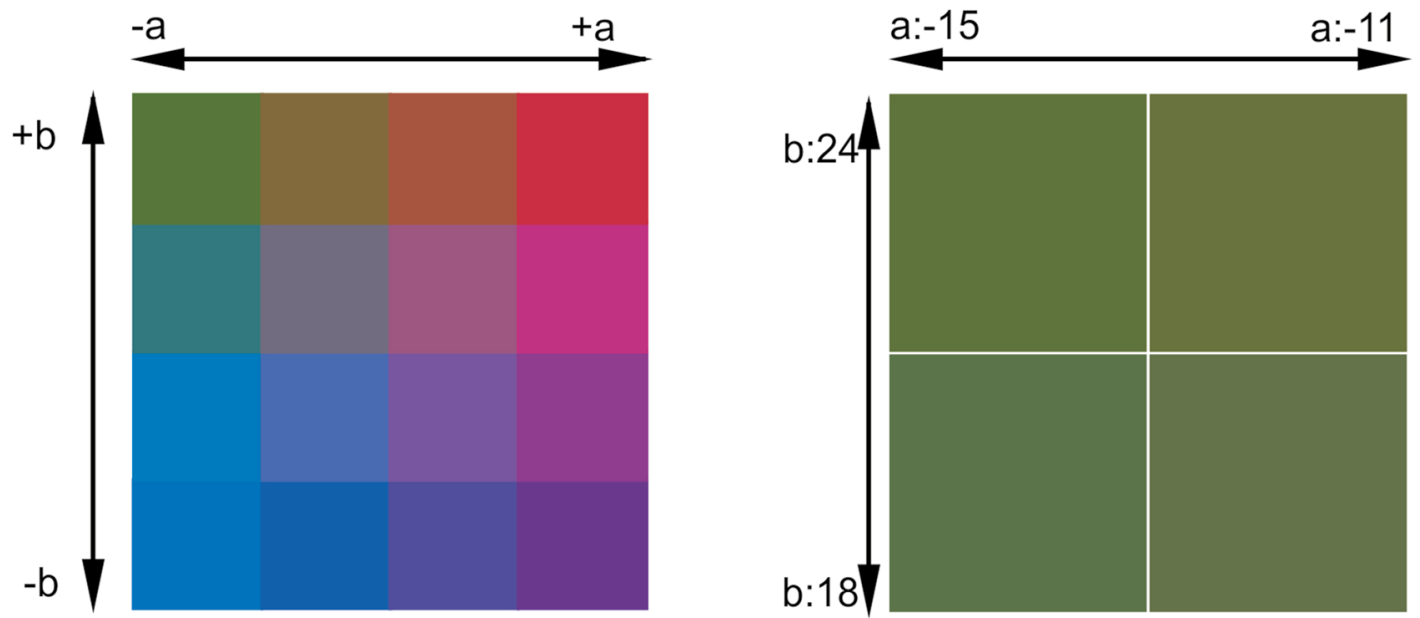

A

Fig. 6. Representación del espacio de color para A: coordenadas rojo/verde ("a") de -20 a 55 y amarillo/ azul ("b") de -80 a 25, y luminosidad ("L") de 48; B: rangos de valores de a y b registrados en genotipos pertenecientes a dos poblaciones de Sorghastrum pellitum cultivados en Balcarce, Buenos Aires; L=48.

\section{Discusıón}

Supervivencia al trasplante y establecimiento final

Las plantas de menor tamaño de Sorghastrum pellitum presentaron el mayor porcentaje de mortandad. Ulrich et al. (2011), al evaluar la supervivencia de individuos a campo, detectaron un mayor porcentaje de mortandad sólo en la etapa posterior al trasplante $(21,5 \%)$ que además era variable en función del tamaño inicial de la mata, es decir, de su número de macollos. Estos resultados coinciden con lo registrado en el presente trabajo ya que se observó que, en la E2, al estar las plantas más desarrolladas, la supervivencia fue mayor que en la E1. Por otro lado, si bien ambas estaciones de crecimiento presentaron diferencias en la cantidad de precipitaciones acumuladas y en su distribución, esta condición no tendría un efecto de mayor relevancia en la supervivencia ya que, en la época más cálida de ambas estaciones, se complementó el agua de lluvia con la de riego. En relación a esto, Ulrich et al. (2011) registraron un buen desarrollo y crecimiento de las plantas aún con niveles de evapotraspiración elevados y balance hídrico negativo. Esta especie mostraría entonces una buena tolerancia a balance hídrico negativo, que puede ser complementado con prácticas de manejo habituales para plantas ornamentales.
Con respecto a las condiciones térmicas, esta variable ambiental no habría afectado la mortandad para Sorghastrum pellitum en este trabajo, dado que la temperatura media fue algo inferior en los meses del período invernal de la E2 con respecto a la E1, sin embargo, el porcentaje de supervivencia de plantas fue superior. Esta respuesta es diferente a lo descrito en la literatura para $S$. pellitum y otras gramíneas estivales, en que se ha reportado que bajas temperaturas, cercanas a $0^{\circ} \mathrm{C}$, pueden disminuir el porcentaje de supervivencia de plantas (Ulrich et al. 2011). Por otro lado, en la E1 las plantas presentaban un menor desarrollo y esta condición pudo haber afectado su supervivencia debido a su menor capacidad, por disponibilidad de recursos propios, para afrontar el período de heladas. Para otras gramíneas estivales, Ries \& Svejcar (1991) encontraron una relación directa entre el número de macollos y la supervivencia en el invierno y mencionan que para garantizar dicha supervivencia las plantas deberían llegar al invierno con al menos un macollo. Por último, en un estudio demográfico de Festuca gracillinia Rothrn., gramínea perenne estival, Oliva et al. (2005) encontraron que la mayor mortandad ocurría en aquellas plantas que presentaban matas pequeñas y medianas. Si bien esto explicaría las diferencias entre la E1 y la E2, las variaciones entre los 
tratamientos estarían relacionadas al efecto de cada genotipo y de las interacciones entre estos y el ambiente. Esto último podría constituir una ventaja al momento de seleccionar el material genético para su mejoramiento o para su aprovechamiento directo con fines ornamentales.

\section{Fenología e intensidad de la floración}

Ambas poblaciones de Sorghastrum pellitum presentaron plantas con flores en las dos estaciones de crecimiento bajo estudio por lo que se pone en evidencia que las señales exógenas y endógenas para que se desencadene la floración (Blázquez \& Weigel, 2000; Blázquez et al., 2001) resultaron favorables, y que los distintos nodos de la cascada de regulación génica de la floración siguieron un curso satisfactorio (Blázquez et al., 2001; Boss et al., 2004, Krizek \& Fletcher, 2005; Soltis et al., 2007). Sin embargo, también se observó que en la E1 hubo tres genotipos de la población BAR para los que no floreció ninguna planta y para los que en la E2 la floración fue muy corta. Es importante tener en cuenta estas diferencias entre genotipos ya que posibilitan la selección de germoplasma con valor ornamental. Por otro lado, también se observaron variaciones intra-genotipo, ya que, en ambas estaciones, dentro de los genotipos que florecieron hubo plantas que no emitieron ninguna panoja. Es posible que dichas plantas hayan sido afectadas por características microambientales a nivel de maceta, desventajosas para la floración, tales como temperatura inadecuada o propiedades nutricionales o hídricas del sustrato deficientes.

Independientemente de la población considerada, el mayor porcentaje de plantas en flor se registró en la segunda estación de crecimiento. A mayor edad de las plantas se espera mejor aclimatación de las mismas al entorno, así como un aumento en el tamaño de los órganos de reserva y por ende en contenido de fotoasimilados (ej. sacarosa), determinando mayor capacidad de las plantas para responder a las señales inductoras de la floración (Blázquez et al., 2001, Eriksson et al., 2006). Por lo tanto, esto podría estar vinculado con la aceleración de la floración, el aumento en el porcentaje de plantas florecidas y al número de panojas por planta registrados en la E2.

La duración de la floración resultó variable a nivel inter- e intra-poblacional y también a nivel intra-genotipo, llegando a extenderse en algunos casos hasta cerca de 40 días. También se observó que el período de floración se extendió a medida que aumentó la edad, lo que se reflejó en la E2 donde los ejemplares eran más adultos y presentaban en general mayor número de varas florales. Esto es una característica de importancia ornamental, ya que se desea que la floración se extienda el mayor tiempo posible. Resulta importante mencionar que algunos de los genotipos analizados fueron descriptos como de floración de sólo un día, lo que los situaría como ejemplares de floración efímera. Sin embargo, esto no fue así ya que en realidad en dichos casos ocurrió que sólo se registró la aparición de una o varias panojas en una sola fecha, pero que se mantuvieron en pie varios días. En trabajos futuros de caracterización de esta especie con fines ornamentales sería importante tener en cuenta esta situación para tener mayor certeza sobre la duración de la panoja en pie.

El inicio de la floración parece tener variabilidad entre poblaciones de esta especie. Para el sudeste bonaerense, Alonso (2005) menciona que Sorghastrum pellitum inicia el rebrote en primavera y produce la elongación del tallo a fin de octubre, vegetando el resto del año, sin embargo, en este ensayo se observó que el período de floración se inició más tarde, específicamente a fines de noviembre, y que se extendió hasta principios de enero, concentrándose en el mes de diciembre. Cabe destacar que los materiales analizados en cada estudio tenían diferentes orígenes siendo los de Alonso (2005) de Sierra de los Padres, una porción aún más austral del Sistema serrano de Tandilia, en el Partido de General Pueyrredón (Provincia de Buenos Aires). Esto, sumado a condiciones ambientales diferenciales y/o metodológicas en cada ensayo podría contribuir a explicar, al menos en parte, las sutiles diferencias en el inicio de la etapa reproductiva en cada estudio.

\section{Caracteres morfológicos}

En cuanto a las características morfológicas evaluadas, se observó que algunos de los genotipos poseen valores que caen dentro de los rangos esperados para la especie, mientras que para otros se exceden esos rangos. Sorghastrum pellitum, según Morrone \& Zuloaga (2012), se caracteriza por formar matas grandes y densas, alcanzando una altura total (incluyendo las cañas) de entre 0,4 a $1,2 \mathrm{~m}$. De acuerdo a lo observado en este ensayo, 


\section{L. Echeverría et al. - Caracterización fenotípica de Sorghastrum pellitum (Poaceae)}

la altura total de las plantas, es decir, la longitud del conjunto dado por la mata, el despeje de la inflorescencia y la panoja fue cercana a los $70 \mathrm{~cm}$, por lo que caería dentro de los rangos citados en la bibliografía.

Con respecto a la longitud de las hojas, Dávila (1988) y Morrone \& Zuloaga (2012) mencionan que Sorghastrum pellitum posee láminas de 15 a 40 (-60) $\mathrm{cm}$ de largo mientras que Cabrera (1970) mencionan que los valores de longitud foliar oscilan entre los 8 y los $20 \mathrm{~cm}$. En este trabajo se observó que la media general de $\mathrm{LF}$ fue de $16,1 \mathrm{~cm}$ y que el rango de esta variable, de $10,6 \mathrm{~cm}$ a $26,2 \mathrm{~cm}$, incluso con variaciones a nivel intra-genotipo. Por lo tanto, estos resultados reflejan valores inferiores a los descriptos por Dávila (1988) y Morrone \& Zuloaga (2012) y superiores a los de Cabrera (1970). Si se tienen en consideración los valores mínimos y máximos de longitud foliar reportados por dichos autores, es decir, de $8 \mathrm{~cm}$ a $40 \mathrm{~cm}$ (excepcionalmente de hasta $60 \mathrm{~cm}$ ), los resultados obtenidos en este trabajo quedan comprendidos dentro de dicho rango y ponen de manifiesto la variabilidad de este carácter.

Muchas especies perennes de esta familia son cespitosas, emitiendo progresivamente macollos que, llegado el período reproductivo, no florecen todos simultáneamente, sino que algunos permanecen en estado vegetativo y pasan a la próxima estación de crecimiento. De esa manera la mata adquiere mayor tamaño a mayor edad de la planta (Cabrera, 1970). Ejemplos de esto lo constituyen gramíneas del género Bromus (Leofanti, 2010) o Saccharum officinarum (Mariotti \& Collavino, 2014), por citar algunos, y también lo constituiría Sorghastrum pellitum ya que, de acuerdo a los resultados de este trabajo, con el correr del tiempo se registraron incrementos en el tamaño de las matas dados por aumentos significativos en el DM y en la AM. A su vez, Alonso (2005) al caracterizar en el SE bonaerense diez plantas de esta especie, procedentes de Sierra de los Padres, encontró que el crecimiento de las matas era escaso en invierno, pero profuso en primavera. Por su parte, Cano (1988 citado en Ulrich et al., 2011) menciona que esta especie inicia su rebrote en octubre-noviembre, florece y fructifica en diciembre y vegeta el resto del año. Esto coincide con lo detectado en este trabajo ya que los aumentos significativos en el DM y AM ocurrieron en las últimas fechas de muestreo, realizadas a finales de la estación primaveral e inicios de la estival.

En paisajismo, la textura hace referencia al tamaño o ancho de las hojas que conforman el follaje (Bellon, 1976). En particular en Gramíneas ornamentales, Rúgolo de Agrasar \& Puglia (2004) sugieren que las especies que poseen un ancho foliar comprendido entre 3 y $10 \mathrm{~mm}$ son de textura media, mientras que valores inferiores $o$ superiores al rango mencionado indican texturas finas o gruesas, respectivamente. Para la variable AF, Sorghastrum pellitum presenta hojas con láminas de 3 a $5 \mathrm{~mm}$ de ancho según Cabrera (1970), es decir, de textura media según Rúgolo de Agrasar \& Puglia (2004), mientras que Dávila (1988) y Morrone \& Zuloaga (2012) reportan valores de ancho foliar de 1 a $3(-5) \mathrm{mm}$, lo que permitiría caracterizar al follaje de esta especie como de textura fina, excepcionalmente de textura media. En el presente trabajo se registró un valor promedio de $\mathrm{AF}$ de $4,7 \mathrm{~mm}$, con un rango comprendido entre 3 y $7 \mathrm{~mm}$ e inclusive con variaciones dentro de un mismo genotipo, con lo cual las hojas de algunas plantas analizadas presentaron textura de fina, mientras que otras, textura media. A su vez, los máximos valores de AF reportados en este trabajo sobre plantas vivas de $S$. pellitum fueron superiores a los citados por los autores antes mencionados, quienes emplearon ejemplares herborizados en sus descripciones. Esto podría causar que las láminas hayan reducido su tamaño tras la desecación propia del proceso de herborización. A pesar de esto, la diferencia en ancho foliar detectada podría estar indicando la existencia de plantas de $S$. pellitum de hojas más anchas a lo reportado. Esta especie presenta variabilidad para el carácter AF que resulta valiosa para realizar actividades de selección, al igual que se ha corroborado en otras especies de Gramíneas estudiadas con fines ornamentales en el SE bonaerense, como Poa iridifolia y Pennisetum spp. (Echeverría et al., 2004; Alonso et al., 2005a, b; Alonso et al., 2011; Pontaroli, 2012; Palmieri, 2013).

Gracias al despeje de las inflorescencias registrado, las plantas de Sorghastrum pellitum poseen la capacidad de generar efecto de transparencia, es decir, el efecto visual que se produce en las plantas cuando son atravesadas por 
la luz natural, permitiendo ver parcialmente los objetos que se encuentran detrás de ellas (Nizzero, 2006). Esta importante característica ornamental pudo ser revelada para esta especie a partir del presente trabajo.

En cuanto a la longitud de la panoja, según Hackel (1883), la misma varía entre 18 y $24 \mathrm{~cm}$; Cabrera (1970), por su parte, reporta variaciones de entre 10 y $30 \mathrm{~cm}$, mientras que Morrone \& Zuloaga (2012) de entre 15 y $30(-40) \mathrm{cm}$. En este trabajo, la LP varió de 8 a $17,5 \mathrm{~cm}$, por lo que los valores mínimos registrados en el presente trabajo están por debajo de los publicados. Por su parte, para la variable AP, los valores registrados fueron también variables, comprendidos entre 0,1 a $4 \mathrm{~cm}$. No se encontraron registros en la bibliografía sobre este carácter por lo que, en base a los resultados obtenidos en este estudio se podría inferir a priori que las panojas de esta especie son delgadas.

En cuanto al color de las hojas, se registraron colores no descritos anteriormente y diferencias significativas entre genotipos. Sorghastrum pellitum ha sido descrita como una especie de láminas glaucas (Hackel, 1883), aunque Alonso (com. pers.) ha observado a las plantas del SE bonaerense como de coloración uniforme verde a verde-grisáceo. Sin embargo, en los materiales analizados predominó el verde oscuro gracias a la preponderancia de los valores negativos del parámetro de color "a" y de los positivos del parámetro " $b$ ", a la vez que resultaron carentes de brillo por el valor intermedio de L. Para esta variable se detectaron diferencias significativas entre genotipos para los parámetros "a" y "b" que podrían ser útiles en la selección de germoplasma con fines ornamentales. Cabe mencionar que según Cabrera (1970), las láminas de $S$. pellitum son áspero pubescentes o algo velludas en la parte inferior, mientras que Morrone \& Zuloaga (2012) las describen como comúnmente pilosas, en ocasiones glabras. Todas las plantas analizadas en este estudio presentaron láminas cubiertas de pelos sedosos, pero no se realizaron análisis para constatar el grado de pubescencia. La pilosidad de las hojas podría generar variaciones en la coloración del follaje, por lo que en estudios futuros de caracterización de esta especie con fines ornamentales podría contemplarse el grado de pubescencia de las láminas como una variable a analizar por su potencial influencia sobre el color de las plantas.
Existe también variabilidad en el color de los pedúnculos de las inflorescencias y en las vainas para esta especie. Las inflorescencias de Sorghastrum pellitum son panojas castaño-rojizas (Morrone \& Zuloaga, 2012) que presentan espiguillas dispuestas sobre pedúnculos rojizos muy llamativos (Alonso, com. pers.). En este trabajo se observó que el color de los pedúnculos fue anaranjado o violáceo y además que esta variable presentó variación dentro de una misma planta. Esto último podría estar indicando que el carácter tendría expresión de tipo cuantitativa, marcadamente influenciada por el ambiente, quizás influido por las temperaturas más bajas de la E2 lo que genera variaciones fenotípicas continuas (Falconer, 1986; Fehr, 1987). Algo similar sucedió con el carácter antocianinas en vaina ya que a pesar de que en general predominó su ausencia, fue variable incluso entre clones pertenecientes al mismo genotipo.

Considerando que muchos de los atributos detectados en Sorghastrum pellitum a lo largo de este trabajo han sido mencionados por Rúgolo de Agrasar \& Puglia (2004) como atractivos en las Gramíneas ornamentales (como efecto de transparencia, inflorescencias coloridas, diferentes texturas en las hojas), los materiales estudiados poseerían cualidades de interés para espacios verdes diseñados. Los datos recabados brindan información que da cuenta de la posibilidad de cultivo de $S$. pellitum, así como también de su fenología y algunos atributos morfológicos que permitirían su eventual incorporación en el mercado floricultor. De esta forma, podría utilizarse en planteos paisajísticos bajo una amplia gama de situaciones, en forma aislada o bien en grupo para potenciar el efecto decorativo. Además, aprovechando su atractivo durante la floración podría emplearse como punto focal o, gracias a su follaje perenne, como planta estructural, formando macizos, borduras, integrando jardines ecológicos, de rocallas o de grava, tanto en grandes extensiones como en jardines pequeños, terrazas o balcones, siempre a pleno sol, en suelo directo o en macetas. Dado el color neutro de su follaje, podría fácilmente combinarse con plantas de colores distintos o bien con otras de colores similares, pero de diferente textura, para realzar la composición visual.

La variabilidad en los caracteres evaluados de Sorghastrum pellitum fue detectada en los niveles inter- e intra-poblacional así como a 


\section{L. Echeverría et al. - Caracterización fenotípica de Sorghastrum pellitum (Poaceae)}

nivel intra-genotipos. Esta variabilidad resulta importante por diversos motivos. Por un lado, algunos de los caracteres registrados no coinciden con lo reportado por Hackel (1883), Cabrera (1970), Dávila (1988) y Zuloaga \& Morrone (2012). A pesar de que en dichas descripciones no se especifica el modo en que se tomaron las mediciones de cada órgano descripto ni el estadio fenológico de las plantas al hacerlo, las mismas surgen de registros efectuados en ejemplares de procedencias disímiles, probablemente en varios órganos y en estadios fenológicos distintos a fin de poder registrar las variaciones en cada carácter. Las discrepancias morfológicas detectadas ponen de relieve la importancia de analizar un gran número de ejemplares de esta especie, y en particular de distintas áreas de su distribución abarcando la totalidad de la misma a la hora de realizar descripciones taxonómicas. En este sentido, consideramos necesario incluir en las descripciones a individuos de poblaciones que prosperan en áreas marginales de su distribución y que, por lo tanto, podrían estar adaptados a condiciones ambientales diferentes a los de la zona central de su distribución, afectando sus características morfológicas.

Por otra parte, se podría considerar también la posibilidad de la presencia de otras entidades taxonómicas para este género. En la revisión sistemática de Sorghastrum, Dávila (1988) sugirió la posible hibridación de $S$. pellitum con $S$. viride, especie que presenta hojas e inflorescencias de mayor tamaño que la primera. Según las observaciones de este autor basadas en ejemplares de herbario, en el área de superposición de ambas especies hay diferentes variantes, algunas morfológicamente más similares a $S$. pellitum y otras a $S$. viride. A pesar de que no hay registros de que $S$. viride prospere en el SE bonaerense, si existieran híbridos que se hubieran dispersado hacia el sur, estos podrían explicar algunas de las diferencias morfológicas encontradas en las plantas analizadas en este estudio con respecto a lo que figura en la bibliografía para $S$. pellitum. Dado que la hibridación entre estas dos especies no ha sido corroborada, la realización de estudios de biología reproductiva con cruzamientos inter-específicos y análisis de la descendencia, entre otros, podrían poner a prueba esta hipótesis.

Finalmente, la variabilidad detectada resulta un antecedente fundamental al momento de tomar decisiones vinculadas a la inclusión de $S$. pellitum en programas de mejoramiento genético. Teniendo en cuenta que la variabilidad representa el insumo básico necesario para la realización de actividades de selección y/o mejoramiento (Rimieri, 2017), estos resultados son alentadores y permiten considerar al germoplasma de esta especie en la obtención de variedades comerciales.

\section{Contribución DE LOS AUTORES}

MLE y GAL diseñaron el trabajo, coleccionaron el material vegetal, tomaron fotografías, tomaron registros y mediciones a campo, realizaron análisis de los datos y redactaron el manuscrito; GES tomó registros y mediciones a campo, realizó análisis de datos y colaboró en la redacción del manuscrito; MME colaboró en el diseño del trabajo, en la colección del material vegetal y en la redacción del manuscrito.

\section{Agradecimientos}

Las autoras agradecen a la Universidad Nacional de Mar del Plata (AGR 557/18 y AGR 616/20, responsable de los subsidios María Mercedes Echeverría) por el financiamiento y a la EEA INTA Balcarce por facilitar las instalaciones para realizar el ensayo a campo. También agradecen a la Ing. Agr. (M. Sc.) Sara I. Alonso por sus valiosos aportes en relación a las características morfológicas de Sorghastrum pellitum. Por último, agradecen a 1@s revisor@s del trabajo, cuyas observaciones contribuyeron a mejorarlo sustancialmente, y a la Editora que amable y solícitamente atendió nuestras consultas.

\section{Bibliografía}

ALONSO, S. I., F.A. VORUSSO \& E.O. MONZÓN. 2004. Crecimiento otoñal de Sorghastrum pellitum en el Sudeste de la provincia de Buenos Aires. Actas de la II Reunión Binacional de Ecología. XXI Reunión Argentina Ecología. XI Reunión de la Sociedad de Ecología de Chile. "Ecología en tiempos de cambio". Santiago de Chile, Chile. p. 293. 
ALONSO, S.I. 2005. Morfogénesis foliar, evolución y estructura de plantas de Sorghastrum pellitum en el SE Bonaerense. Rev Arg. Producción Animal. 25: 141-142.

ALONSO, S.I.; M.L. ECHEVERRÍA, M.F. GENOVESE, M.G. MONTERUBBIANESI \& A.C. RIDAO. 2005a. Germoplasma de Poa iridifolia Hauman: Tamaño foliar e incidencia de Roya. Actas de las XXX Jornadas Argentinas de Botánica. Rosario, Argentina. p. 197.

ALONSO, S.I., M. DIAZ, M.L. ECHEVERRÍA, M.F. GENOVESE \& M.P PERMINGEAT. 2005b. Variabilidad en color del follaje en poblaciones de Poa iridifolia. Actas del V SIRGEALC. Montevideo, Uruguay. p. 37.

ALONSO, S.I., M.L. ECHEVERRÍA \& A.E. LUCHINI. 2011. Nivel de ploidía y tamaño de matas y láminas en clones de Pennisetum alopecuroides. Actas del VIII Simposio de Recursos Genéticos para América Latina y el Caribe. Quito, Ecuador.

BELLÓN, C. A. 1976. Fundamentos del planeamiento paisajista. Enciclopedia Argentina de Agricultura y Jardinería. Tomo II. Acme, Buenos Aires.

BENTON, T. G., J.A. VICKERY \& J.D. WILSON. 2003. Farmland biodiversity: is habitat heterogeneity the key? Tree 18: 182-188.

https://doi.org/10.1016/S0169-5347(03)00011-9

BLÁZQUEZ, M. \& D. WEIGEL. 2000. Integration of floral inductive signals in Arabidopsis. Nature. 404: 889-892. https://doi.org/10.1038/35009125

BLÁZQUEZ, M., M. KOORNNEEF \& J. PUTTERILL. 2001. Flowering on time: genes that regulate the floral transition. EMBO Rep. 12: 1078-1082. http://doig.org/10.1093/embo-reports/kve254

BOSS, P.K., R.M. BASTOW, J.S. MYLNE \& C. DEAN. 2004. Multiple pathways in the decision to flower: enabling, promoting, and resetting. Plant Cell. 16: 18-31. https://dx.doi.org/10.1105\%2Ftpc.015958

BROOKES, J. 2013. Guía completa de diseño de jardines. Blume, Barcelona.

BURGUEÑO, G. \& C. NARDINI. 2018. Elementos de diseño y planificación con plantas nativas. Introducción al Paisaje Natural. Parte I. Generalidades. Orientación Gráfica Editora, Buenos Aires.

CABRERA, A. 1970. Flora de la Provincia de Buenos Aires. Tomo IV, parte II. Gramíneas. Colección científica del INTA, Buenos Aires.

CLAYTON, W.D., M.S. VORONTSOVA, K.T. HARMAN \& H. WILLIAMSON. 2021. GrassBase-
The Online World Grass Flora [online]. Disponible en: http://www.kew.org/data/grasses-db.html. [Acceso: 30 junio 2021].

DÁVILA, A.P.D. 1988. Systematic revision of the genus Sorghastrum (Poaceae: Andropogoneae). Tesis doctoral. Iowa State University, Estados Unidos.

DI RIENZO, J.A., F. CASANOVES, M.G. BALZARINI, L. GONZALEZ, M. TABLADA \& C.W. ROBLEDO. 2016. InfoStat versión 2016. Grupo InfoStat, FCA, Universidad Nacional de Córdoba, Argentina. Disponible en: http://www.infostat.com.ar .

EEA Balcarce INTA. 2021. Información Agrometeorológica. [on line]. Disponible en: http://www.inta.gov.ar/balcarce/info/meteoro.html [Acceso: 1 junio 2021].

ECHEVERRÍA, M.L., M.F. GENOVESE, A.E. LUCHINI \& S.I. ALONSO. 2004. Variabilidad en longitud y ancho foliar otoñal en poblaciones de Poa iridifolia. Actas de la II Reunión Binacional de Ecología, Ecología en tiempos de cambio. Mendoza, Argentina. 453 p.

ECHEVERRÍA, M.L., S.I. ALONSO \& V.M. COMPARATORE. 2017. Survey of the vascular plants of Sierra Chica, the untouched area of the Paititi Natural Reserve (southeastern Tandilia mountain range, Buenos Aires province, Argentina). Check List. 13: 1003-1036.

https://doi.org/10.15560/13.6.1003

ECHEVERRÍA, M.L. 2018. Caracterización fenotipica, citogenética y molecular de poblaciones silvestres de Chrysolaena flexuosa (Sims) H. Rob. en el sudeste bonaerense. Tesis doctoral. Universidad Nacional de Mar del Plata, Argentina.

ECHEVERRÍA, M.L \& E.L. CAMADRO. 2020. Morphological and molecular variability of wild diploid and polyploid populations of Chrysolaena flexuosa (Sims) H. Rob.: relevance for ornamental breeding. Sci. Hortic. 260: 108875.

https://doi.org/10.1016/j.scienta.2019.108875

ERIKSSON, S., H. BÖHLENIUS, T. MORITZ \& O. NILSSON. 2006. GA4 Is the Active Gibberellin in the Regulation of LEAFY Transcription and Arabidopsis Floral Initiation. Plant Cell 18: 21722181. https://doi.org/10.1105/tpc.106.042317

EYNARD, C., A. CALVIÑO \& L. ASHWORTH. 2020. Cultivo de plantas nativas. Propagación y viverismo de especies de Argentina central. Ecoval Ediciones, Córdoba.

FALCONER, D.E. 1986. Introducción a la genética cuantitativa. Cecsa, México D.F. 


\section{L. Echeverría et al. - Caracterización fenotípica de Sorghastrum pellitum (Poaceae)}

FACCIUTO, G., P. BOLOGNA, V. BUGALlO \& M.C. RIVERA. 2019. Recursos Genéticos Ornamentales Nativos de Argentina: de la colecta a la producción. Rev $R G$ News. 5: 13-17. http://hdl.handle. net/20.500.12123/4798

FEHR, W.R. 1987. Principles of cultivar development: theory and technique Vol I. Macmillan, New York.

FORD-LLOYD, B. \& M. JACKSON. 1986. Plant genetic resources: an introduction to their conservation and use. Edward Arnold, London.

GODDARD, M. A., A.J. DOUGIL \& T.G. BENTON. 2010. Scaling up from gardens: biodiversity conservation in urban environments. Trends in Ecol \& Evolution. 25: 90-98.

https://doi.org/10.1016/j.tree.2009.07.016

HACKEL, E. 1883. Gramineae. En C.F.P. VON MARTIUS (ed.) Flora Brasiliensis. Vol. 2(3): 273277. Munich, Vienna. [online]. Disponible en: https:// www.biodiversitylibrary.org/item/9651\#page/5/ mode/1up [Acceso: 22 septiembre 2021].

KRIZEK, B.A. \& FLETCHER, J.C. 2005. Molecular mechanisms of flower development: an armchair guide. NAT. REV. GENET. 6: 688-698. https://doi.org/10.1038/nrg1675

LEOFANTI, G. A. 2010. Evaluación de poblaciones de Bromus spp. (Secc. Ceratochloa). I-Variabilidad en tamaño foliar y número de macollos. Tesis de Ingeniería Agronómica. Universidad Nacional de Mar del Plata, Argentina.

MARIOTTI, J. A. \& N.G. COLLAVINO. 2014. Los caracteres cuantitativos en la mejora genética de los cultivos. Orientación Gráfica Editora, Buenos Aires.

MORRONE O. \& F.O. ZULOAGA. 2012. Sorghastrum Nash. En: ZULOAGA, F.O., Z.E. RÚGOLO \& A.M. ANTON (eds.). Flora Vascular de la República Argentina 3: 551-554. Córdoba: Graficamente Ediciones.

NICORA, E.G. \& Z.E. RÚGOLO DE AGRASAR. 1987. Los géneros de gramíneas de América Austral, Argentina, Chile, Uruguay y áreas limitrofes de Bolivia, Paraguay y Brasil. Hemisferio Sur, Buenos Aires.

NIZZERO, G. 2006. El paisaje ilustrado. Glosario Técnico. Editorial Facultad de Agronomía, Universidad de Buenos Aires, Buenos Aires.

OLIVA, G., M. COLLANTES \& G. HUMANO. 2005. Demography of Grazed Tussock Grass Populations in Patagonia. Rangeland Ecol. Manag. 58: 466-473. https://doi.org/10.2111/1551-5028(2005)58[466:DO GTGP]2.0.CO;2
PALMIERI, R. 2013. Caracterización morfológica y nivel de ploidia en clones de Pennisetum alopecuroides (L.) Spreng y entidades afines. Tesis Ingeniería Agronómica, Universidad Nacional de Mar del Plata, Argentina.

PARODI, L. R. 1930. Ensayo fitogeográfico sobre el partido de Pergamino. Revista Fac. Agron. Veterin. 7:65-271.

PONTAROLI, D. 2012. Fenología, morfología reproductiva y viabilidad de semillas en clones de Pennisetum alopecuroides L. Tesis de Licenciatura en Producción Vegetal. Universidad Nacional de Mar del Plata, Argentina.

RIES, R. E. \& T.J. SVEJCAR. 1991. The grass seedling: When is it established? Rangeland ecol. Manag. 44: 574-576. http://dx.doi.org/10.2307/4003038

RIMIERI, P. 2017. La diversidad genética y la variabilidad genética, dos conceptos diferentes asociados al germoplasma y al mejoramiento genético. J. Basic \& Applied Genetics 28: 7-13. https://sag.org.ar/jbag/wpcontent/uploads/2019/11/A1_7-13-2.pdf

RÚGOLO DE AGRASAR, Z.E. \& M.L. PUGLIA. 2004. Plantas de la Argentina silvestres y cultivadas. Gramíneas ornamentales. Vol. 1. $1^{\text {ra }}$ edición. Editorial L.O.L.A., Buenos Aires.

SOLTIS, D., A.S. CHANDERBALI, K. SANGTAE, M. BUZGO \& P. SOLTIS. 2007. The ABC model and its applicability to basal angiosperms. Ann Bot. London 100: $155-163$.

https://dx.doi.org/10.1093\%2Faob\%2Fmcm 117

THIERS, B. Continuamente actualizado. Index Herbariorum: A global directory of public herbaria and associated staff. New York Botanical Garden's Virtual Herbarium. http://sweetgum.nybg.org/science/ih/

TSCHARNTKE, T., Y. CLOUGH, T.C. WANGER, L. JACKSON, I. MOTZKE, I. PERFECTO, J.VANDERMEER \& A. WHITBREAD. 2012. Global food security, biodiversity conservation and the future of agricultural intensification. Biol. Conserv. 151: 5359. https://doi.org/10.1016/j.biocon.2012.01.068

ULRICH, A., C. CHIRINO \& E.U. MORICI. 2011. Evaluación de una población de Sorghastrum pellitum (Hack.) Parodi hasta su reintroducción en áreas medanosas. Rev. Fac. Agronomía UNLP. 22: 2231. https://cerac.unlpam.edu.ar/index.php/semiarida/ article/view/4523/4685

ZULOAGA, F., M. BELGRANO \& C. ZANOTTI. 2019. Actualización del Catálogo de las Plantas Vasculares del Cono Sur. Darwiniana, Nueva Serie. 7: 208-278. https://doi.org/10.14522/darwiniana.2019.72.861 
